Math. Model. Nat. Phenom.

Vol. 5, No. 4, 2010, pp. 122-149

DOI: $10.1051 / \mathrm{mmnp} / 20105405$

\title{
Wave Equation with Slowly Decaying Potential: asymptotics of Solution and Wave Operators
}

\author{
S. A. Denisov ${ }^{1}$ \\ University of Wisconsin-Madison, Mathematics Department \\ 480 Lincoln Dr., Madison, WI, 53706, USA
}

\begin{abstract}
In this paper, we consider one-dimensional wave equation with real-valued squaresummable potential. We establish the long-time asymptotics of solutions by, first, studying the stationary problem and, second, using the spectral representation for the evolution equation. In particular, we prove that part of the wave travels ballistically if $q \in L^{2}\left(\mathbb{R}^{+}\right)$and this result is sharp.
\end{abstract}

Key words: wave equation, square-summable potential, wave operators

AMS subject classification: $35 \mathrm{~L} 05$

\section{Introduction}

We will consider the Cauchy problem for the wave equation

$$
\begin{array}{r}
y_{t t}(x, t)=y_{x x}(x, t)-q(x) y(x, t), \quad y(x, 0)=\phi(x), \quad y_{t}(x, 0)=\psi(x), \\
y(0, t)=0, \quad t \geq 0, x \in \mathbb{R}^{+}
\end{array}
$$

where $q(x)$ is time-independent, real-valued, and $q(x) \in L^{2}\left(\mathbb{R}^{+}\right)$. In the free case (i.e., when $q=0)$,

$$
y(x, t)=\left(\frac{\phi_{o}(x+t)+\phi_{o}(x-t)}{2}+\frac{1}{2} \int_{x-t}^{x+t} \psi_{o}(s) d s\right) \chi_{x>0}
$$

\footnotetext{
${ }^{1}$ E-mail: denissov@math.wisc.edu
} 
where $\phi_{o}$ and $\psi_{o}$ are odd continuations of $\phi$ and $\psi$ to $\mathbb{R}$. Assuming that $\phi$ and $\psi$ are smooth and compactly supported, this formula gives the classical solution that propagates with finite speed.

If $q \neq 0$, the stationary operator for the problem is one-dimensional Schrödinger

$$
H f=-f^{\prime \prime}+q f, \quad f(0)=0, \quad f \in W^{2,2}\left(\mathbb{R}^{+}\right)
$$

The conservation law is

$$
E(t)=\left\langle y_{t}, y_{t}\right\rangle+\langle H y, y\rangle=\text { const }
$$

In this paper, we will study the long-time behavior of $y(x, t)$ which is the main question of the scattering theory for (1.1). The scattering theory of wave equation is the classical subject and was extensively studied in the literature ([9] is an excellent reading). It is known that the spectrum of stationary operator can be pure point as long as $q \in L^{p}\left(\mathbb{R}^{+}\right)$with $p>2[10,12]$ and that will show the sharpness of our result on the $L^{p}$-scale. The method is a modification of the one employed for Dirac operator [4]. We heavily relied on the "trace identities" obtained in [8]. The paper [8] can also be a source for the proofs of various well-known results we mention in the text. In the context of polynomials orthogonal on the unit circle, some progress was recently made in $[2,5,11]$.

The organization of the paper is as follows: in the second section, we introduce the modified Jost function and obtain its multiplicative representation. In the third section, we prove asymptotics of the generalized eigenfunctions for the stationary Schrödinger operator. In the fourth one, we apply this result to prove theorem on asymptotics of solution to wave equation. For that, we need to additionally assume that potential oscillates in a certain way. The last section contains the proof of the existence of modified wave operators in general case. In the Appendix, we collected some standard results we used in the paper.

We will use the following definition for the (inverse) Fourier transform of the function $f(x)$

$$
\hat{f}(k)=\int_{\mathbb{R}} f(x) e^{i k x} d x
$$

\section{Schrödinger operator with $L^{2}\left(\mathbb{R}^{+}\right)$potential: some prelimi- naries}

\subsection{Modified Jost solutions}

Let $u(x, k)$ be the solution to

$$
-u^{\prime \prime}+q u=E u, \quad u(0, k)=0, u^{\prime}(0, k)=1
$$

In this paper we will always take $E=k^{2}$. Assume that $q \in L^{2}\left(\mathbb{R}^{+}\right)$which by Weyl's theorem implies $\sigma_{\text {ess }}(H)=[0, \infty)$. Denote the spectral measure of $H$ by $d \rho(E)$. We have the following generalized Plancherel identity

$$
\int_{\mathbb{R}}|\breve{f}(E)|^{2} d \rho(E)=\|f\|_{2}^{2}
$$


for any $f \in L^{2}\left(\mathbb{R}^{+}\right)$where

$$
\breve{f}(E)=\int_{0}^{\infty} f(x) u(x, k) d x
$$

and the integral is defined in the $L^{2}$-sense. The case of square summable potential is wellunderstood now, e.g., the criterion for $q \in L^{2}\left(\mathbb{R}^{+}\right)$in terms of $d \rho$ was obtained in [8]. Let us write

$$
d \rho(E)=d \rho_{s}(E)+\mu(E) d E
$$

where $d \rho_{s}$ is singular part and $\mu$ is the density of a.c. part.

We will need to control the asymptotical behavior of $u(x, k)$ for $x \rightarrow+\infty$. The pointwise in $k$ results of that type were obtained in [1] for $q \in L^{p}\left(\mathbb{R}^{+}\right)$with $p<2$. We will obtain the asymptotics of $u(x, k)$ for real $k$ but in the integral sense. To this end, we take $k \in \mathbb{C}^{+}$and define the modified Jost functions as follows.

For arbitrary large $R$, consider the truncation $q_{R}(x)=q(x) \chi_{x<R}$. Now that $q_{R} \in L^{1}\left(\mathbb{R}^{+}\right)$, we can define the usual Jost solution $j(x, k, R)$ by requiring

$$
-j^{\prime \prime}+q_{R} j=k^{2} j, \quad j(x, k, R)=e^{i k x}, x>R
$$

The function $j(x, k, R)$ exists not only for any $k \in \mathbb{C}^{+}$but also for real $k \neq 0$. That, for instance, follows from the integral equations for $j(x, k, R)$. Indeed, rewrite (2.1) as

$$
J^{\prime}=\left[\begin{array}{cc}
0 & 1 \\
q_{R}-k^{2} & 0
\end{array}\right] J, \quad J=\left(\begin{array}{l}
j \\
j^{\prime}
\end{array}\right)
$$

and for

$$
Z=\left[\begin{array}{cc}
e^{i k x} & e^{-i k x} \\
i k e^{i k x} & -i k e^{-i k x}
\end{array}\right]^{-1} J
$$

we have

$$
Z^{\prime}=\frac{i q_{R}}{2 k}\left[\begin{array}{cc}
-1 & -e^{-2 i k x} \\
e^{2 i k x} & 1
\end{array}\right] Z, \quad Z=\left(z_{1}, z_{2}\right)^{t}, \quad Z(R)=(1,0)^{t}
$$

If

$$
Z=\left[\begin{array}{cc}
e^{i \phi} & 0 \\
0 & e^{-i \phi}
\end{array}\right] \Psi, \quad \phi(x, k, R)=(2 k)^{-1} \int_{x}^{R} q(t) d t
$$

we finally get

$$
\Psi^{\prime}=\frac{i q_{R}}{2 k}\left[\begin{array}{cc}
0 & -e^{-2 i k x-2 i \phi(x, k, R)} \\
e^{2 i k x+2 i \phi(x, k, R)} & 0
\end{array}\right] \Psi, \quad \Psi=\left(\psi_{1}, \psi_{2}\right)^{t}
$$

Due to asymptotics of $j$ at infinity, we have the integral equations for $\psi_{1}, \psi_{2}$ :

$$
\begin{array}{r}
\psi_{1}(x, k, R)=1+\frac{i}{2 k} \int_{x}^{\infty} q_{R}(t) e^{-2 i k t-2 i \phi(t, k, R)} \psi_{2}(t, k, R) d t \\
\psi_{2}(x, k, R)=-\frac{i}{2 k} \int_{x}^{\infty} q_{R}(t) e^{2 i k t+2 i \phi(t, k, R)} \psi_{1}(t, k, R) d t
\end{array}
$$


Then, one can easily show that iterations converge for any $k \in \overline{\mathbb{C}^{+}} \backslash\{0\}$. Moreover, if $k \in \mathbb{C}^{+}$, then this convergence is uniform in $R$ since $q \in L^{2}\left(\mathbb{R}^{+}\right)$. The function $j(0, k, R)$ is called the Jost function for $q_{R}$, the truncated $q$. We will denote it by $j(k, R)$. Clearly, we have

$$
\begin{array}{r}
j(k, R)=j(0, k, R)=\exp (i \phi(0, k, R)) \psi_{1}(0, k, R)+\exp (-i \phi(0, k, R)) \psi_{2}(0, k, R)= \\
=\exp (i \phi(0, k, R)) j_{m}(k, R)
\end{array}
$$

where

$$
j_{m}(k, R)=\psi_{1}(0, k, R)+\exp (-2 i \phi(0, k, R)) \psi_{2}(0, k, R)
$$

We will call $j_{m}(k, R)$ the modified Jost function for truncated $q$. The function $j(k, R)$ is analytic in $k$ in $\mathbb{C}^{+}$and, in general, might have zeroes on $i \mathbb{R}^{+}$. If these zeroes are denoted by $\left\{i k_{j}(R)\right\}$, then $\left\{-k_{j}^{2}(R)\right\}$ are the negative eigenvalues of $H_{R}$. Since $j(x, k, R)$ is well-defined for real $k \neq 0$ and the Wronskian $W(j(x, k, R), \overline{j(x, k, R)})=2 i k$, we have

$$
u(x, k)=(2 i k)^{-1}(\overline{j(k, R)} j(x, k, R)-j(k, R) \overline{j(x, k, R)}), \quad x \in[0, R]
$$

In particular, taking $x=R$, we get

$$
\begin{aligned}
& u(R, k)=(2 i k)^{-1}\left(\overline{j(k, R)} e^{i R k}-j(k, R) e^{-i R k}\right)= \\
& =(2 i k)^{-1}\left(\overline{j_{m}(k, R)} e^{i R k-i \phi(0, k, R)}-j_{m}(k, R) e^{-i R k+i \phi(0, k, R)}\right) \\
& k^{2} u(R, k)^{2}+u^{\prime}(R, k)^{2}=|j(k, R)|^{2}=\left|j_{m}(k, R)\right|^{2}
\end{aligned}
$$

Later on, we will need a formula for $\partial_{R} j(k, R)$.

Lemma 1. For any real $k \neq 0$, we have

$$
\partial_{R} j(k, R)=\frac{q(R)}{2 i k}\left(-j(k, R)+\overline{j(k, R)} e^{2 i k R}\right)
$$

Proof. Denote the fundamental matrix for (2.2) by $Y\left(x_{1}, x\right)$ such that

$$
\partial_{x} Y=\frac{i q_{R}(x)}{2 k}\left[\begin{array}{cc}
-1 & -e^{-2 i k x} \\
e^{2 i k x} & 1
\end{array}\right] Y, \quad Y\left(x_{1}, x_{1}\right)=I
$$

Notice that

$$
Y(R, x)=\left(\begin{array}{cc}
z_{1}(x, R) & \bar{z}_{2}(x, R) \\
z_{2}(x, R) & \bar{z}_{1}(x, R)
\end{array}\right)
$$

by (2.2). Write

$$
\begin{array}{r}
z_{1}(x, R)=1+\int_{x}^{R} \frac{i q(t)}{2 k} z_{1}(t, R) d t+\int_{x}^{R} \frac{i q(t)}{2 k} e^{-2 i k t} z_{2}(t, R) d t \\
z_{2}(x, R)=-\int_{x}^{R} \frac{i q(t)}{2 k} e^{2 i k t} z_{1}(t, R) d t-\int_{x}^{R} \frac{i q(t)}{2 k} z_{2}(t, R) d t
\end{array}
$$


for $x<R$. Differentiating in $R$ for fixed $x$,

$$
\begin{array}{r}
\partial_{R} z_{1}(x, R)=\frac{i q(R)}{2 k}+\int_{x}^{R} \frac{i q(t)}{2 k} \partial_{R} z_{1}(t, R) d t+\int_{x}^{R} \frac{i q(t)}{2 k} e^{-2 i k t} \partial_{R} z_{2}(t, R) d t \\
\partial_{R} z_{2}(x, R)=-\frac{i q(R)}{2 k} e^{2 i k R}-\int_{x}^{R} \frac{i q(t)}{2 k} e^{2 i k t} \partial_{R} z_{1}(t, R) d t-\int_{x}^{R} \frac{i q(t)}{2 k} \partial_{R} z_{2}(t, R) d t
\end{array}
$$

and this identity is for $L^{2}$ functions in $R$. Differentiating in $x$, we have

$$
\left(\partial_{R} z_{1}(x, R), \partial_{R} z_{2}(x, R)\right)^{t}=\frac{i q(R)}{2 k} Y(R, x)\left(1,-e^{2 i k R}\right)^{t}
$$

Substitute (2.9) and

$$
z_{1}(0, R)=\frac{1}{2 i k}\left(i k j(0, k, R)+j^{\prime}(0, k, R)\right), \quad z_{2}(0, R)=\frac{1}{2 i k}\left(i k j(0, k, R)-j^{\prime}(0, k, R)\right)
$$

to get (2.8).

Our goal is to prove asymptotics of $j_{m}(k, R)$ as $R \rightarrow \infty$. Having that at hand, we can obtain the asymptotics for $u(R, k)$ by (2.6). At this point, it is crucial that we work with analytic continuation of $j_{m}(k, R)$ to the upper half-plane.

As we mentioned before, one can prove that $j_{m}(k, R) \rightarrow j_{m}(k)$ uniformly over the compacts in $\mathbb{C}^{+}$by working with integral equations (2.4). Instead, one can use the determinantal representation for $j(k, R)$ which was advocated in [7]. It is a rather well-known fact that

$$
j(k, R)=\operatorname{det}\left(\frac{H_{R}-k^{2}}{H^{0}-k^{2}}\right)=\operatorname{det}\left(I+R^{0}\left(k^{2}\right) q_{R}\right)
$$

where $H^{0}=-\partial^{2}$ with Dirichlet condition at zero, $R^{0}(z)=\left(H^{0}-z\right)^{-1}$, and det is the perturbation determinant. The regularization of perturbation determinant gives

$$
\operatorname{det}\left(I+R^{0}\left(k^{2}\right) q_{R}\right)=\exp \left(\operatorname{tr}\left(R^{0}\left(k^{2}\right) q_{R}\right)\right) \operatorname{det}_{2}\left(I+R^{0}\left(k^{2}\right) q_{R}\right)
$$

Since

$$
\left[R^{0} f\right](x)=-\int_{0}^{\infty} \frac{e^{i k|x-y|}-e^{i k(x+y)}}{2 i k} f(y) d y
$$

we obtain

$$
\begin{aligned}
\operatorname{det}(I & \left.+R_{0}\left(k^{2}\right) q_{R}\right)=\exp \left(-\int_{0}^{R} \frac{1-e^{2 i k x}}{2 i k} q(x) d x\right) \operatorname{det}_{2}\left(I+R_{0}\left(k^{2}\right) q_{R}\right)= \\
& =\exp \left(-\frac{1}{2 i k} \int_{0}^{R} q(x) d x\right) \exp \left((2 i k)^{-1} \hat{q}_{R}(2 k)\right) \operatorname{det}_{2}\left(I+R_{0}\left(k^{2}\right) q_{R}\right)
\end{aligned}
$$

where $\hat{q}_{R}(k)$ is the (inverse) Fourier transform of $q_{R}$. By (2.3), (2.5), and (2.10),

$$
j_{m}(k, R)=\exp \left((2 i k)^{-1} \hat{q}_{R}(2 k)\right) \operatorname{det}_{2}\left(I+R_{0}\left(k^{2}\right) q_{R}\right)
$$

so 


\section{Definition 2. (Modified Jost function)}

$$
j_{m}(k, R) \rightarrow j_{m}(k)=\exp \left((2 i k)^{-1} \hat{q}(k)\right) \operatorname{det}_{2}\left(I+R_{0}\left(k^{2}\right) q\right)
$$

and the convergence is uniform over the compacts in $\mathbb{C}^{+}$due to $q \in L^{2}\left(\mathbb{R}^{+}\right)$and the standard properties of the regularized determinants.

For the Weyl-Titchmarsh function $m_{R}(z)$ of truncated potential, we have two different representation. One is through the spectral measure

$$
m_{R}(z)=\int\left(\frac{1}{E-z}-\frac{E}{1+E^{2}}\right) d \rho_{R}(E)+C_{R}, \quad \int_{\mathbb{R}} \frac{d \rho_{R}(E)}{1+E^{2}} d E<C
$$

and the other is through the so-called Weyl's solution, which in our case is a multiple of the Jost solution, so

$$
m_{R}(z)=\frac{j^{\prime}(0, k, R)}{j(0, k, R)}, \quad z=k^{2}
$$

Since $q_{R}$ is compactly supported, $d \rho_{R}$ is absolutely continuous on $\mathbb{R}^{+}$, and $j(0, k, R)$ is continuous up to the real line, we have

$$
\pi \mu_{R}(E)=\frac{1}{2 i}\left(\frac{j^{\prime} \bar{j}-\overline{j^{\prime}} j}{|j|^{2}}\right)=k|j(k, R)|^{-2}
$$

In the next section, we will show that the same factorization identity holds for $j_{m}(k)$ and $\mu(E)$.

\subsection{The $a(k)$-coefficient and its modification}

Take the truncated potential $q_{R}$, continue it to $\mathbb{R}^{-}$by zero, and consider the Schrödinger equation on the line. Then, for the Jost solution, we get

$$
j(x, k, R)=a(k, R) e^{i k x}+b(k, R) e^{-i k x}, \quad x<0
$$

The functions $a$ and $b$ possess many interesting properties that we list below:

1. The identity $|a(k, R)|^{2}=1+|b(k, R)|^{2}$ is true for any $k \in \mathbb{R} \backslash\{0\}$. Consequently, $|a(k, R)| \geq$ 1 on the real line. Also, $a(-k, R)=\overline{a(k, R)}$ so $|a(k, R)|$ is even.

2. The function $a(k, R)$ is analytic in $k \in \mathbb{C}^{+}$and is continuous up to $\mathbb{R} \backslash\{0\}$. It can have zeroes only on $i \mathbb{R}^{+}$. If they are denoted by $\left\{i \xi_{j}(R)\right\}$, then the negative eigenvalues of the operator

$$
-\frac{d^{2}}{d x^{2}}+q_{R} \chi_{x>0}, \quad x \in \mathbb{R}
$$

are exactly $\left\{-\xi_{j}^{2}(R)\right\}$. 
3. The functions $m, j, a$, and $b$ are related to each other by

$$
\begin{gathered}
j(k, R)=a(k, R)+b(k, R), \quad m_{R}\left(k^{2}\right)=i k \frac{a(k, R)-b(k, R)}{a(k, R)+b(k, R)} \\
a(k, R)=\left(\frac{m_{R}\left(k^{2}\right)+i k}{2 i k}\right) j(k, R)
\end{gathered}
$$

By (2.5) and (2.13), we have

$$
a(k, R)=\exp \left(\frac{i}{2 k} \int_{0}^{\infty} q_{R}(x) d x\right) a_{m}(k, R), \quad a_{m}(k, R)=\left(\frac{m_{R}\left(k^{2}\right)+i k}{2 i k}\right) j_{m}(k, R)
$$

so $a_{m}$ and $a$ are different only by a singular function that (in its multiplicative representation) corresponds to a jump at zero with the mass

$$
\sim \int_{0}^{\infty} q_{R}(x) d x
$$

which can not be controlled as $R \rightarrow \infty$.

4. We have

$$
a_{m}(k, R)=1-\frac{1}{8 i k^{3}} \int_{0}^{\infty} q_{R}^{2}(x) d x+\bar{o}\left(k^{-3}\right)
$$

as $\Im k \geq 0$ and $|k| \rightarrow \infty$ (indeed, that can be easily observed since $a_{m}(k, R)=\psi_{1}(0, k, R)$ due to (2.4)).

Definition 3. (Modified $a(k)$-coefficient)

$$
a_{m}(k, R) \rightarrow a_{m}(k)=\left(\frac{m\left(k^{2}\right)+i k}{2 i k}\right) j_{m}(k), \quad k \in \mathbb{C}^{+}
$$

Similarly to (2.14),

$$
a_{m}(k)=1-\frac{1}{8 i k^{3}} \int_{0}^{\infty} q^{2}(x) d x+\bar{o}\left(k^{-3}\right), \quad \Im k \rightarrow+\infty
$$

One can easily show that $a_{m}(k)$ is analytic in $\mathbb{C}^{+}$. If its zeroes are denoted by $\left\{i \xi_{j}\right\}$, then the negative eigenvalues of

$$
H_{l}=-\frac{d^{2}}{d x^{2}}+q \chi_{x>0}, \quad x \in \mathbb{R}
$$

are exactly $\left\{-\xi_{j}^{2}\right\}$. 
Next, let us obtain the integral representation for $a_{m}(k, R)$ and $a_{m}(k)$. We have

$$
a(k, R)=B(k, R) \exp \left(\frac{1}{i \pi} \int_{\mathbb{R}} \frac{1+t k}{(t-k)\left(1+t^{2}\right)} \ln |a(t, R)| d t\right)
$$

and $B(k, R)$ is the standard Blaschke product

$$
B(k, R)=\prod_{j} \frac{k-i \xi_{j}(R)}{k+i \xi_{j}(R)}
$$

Taking $k=i y, y \rightarrow+\infty$ we have

$$
\prod_{j} \frac{y-\xi_{j}(R)}{y+\xi_{j}(R)} \exp \left(\frac{1}{\pi} \int_{\mathbb{R}} \frac{y}{t^{2}+y^{2}} \ln |a(t, R)| d t\right) \sim 1+\frac{1}{2 y} \int_{0}^{\infty} q_{R}(x) d x
$$

Since $|a(t, R)| \geq 1$ for real $t$,

$$
\int_{\mathbb{R}} \ln |a(t, R)| d t=\frac{\pi}{2} \int_{0}^{\infty} q_{R}(x) d x+2 \pi \sum_{j} \xi_{j}(R)
$$

and, since $|a(t, R)|$ is even in $t$,

$$
a_{m}(k, R)=B_{m}(k, R) \exp \left(\frac{1}{\pi i} \int_{\mathbb{R}} K(t, k) \ln \left|a_{m}(t, R)\right| d t\right)
$$

where

$$
K(t, k)=\frac{t^{2}}{k^{2}(t-k)}
$$

is a modified Cauchy kernel. For the modified Blaschke product, we get the formula

$$
B_{m}(k, R)=\prod_{j}\left(\frac{k-i \xi_{j}(R)}{k+i \xi_{j}(R)} \exp \left(2 i k^{-1} \xi_{j}(R)\right)\right)
$$

We can now rewrite (2.16) as

$$
a_{m}(k, R)=B_{m}(k, R) \exp \left(\frac{1}{2 \pi i} \int_{\mathbb{R}} K(t, k) \ln \left(\frac{\left|m_{R}\left(t^{2}\right)+i t\right|^{2}}{4 \pi|t| \mu_{R}\left(t^{2}\right)}\right) d t\right)
$$

By [8], formula (1.33), we have

$$
\frac{2}{3} \sum_{j} \xi_{j}^{3}+\frac{1}{\pi} \int_{0}^{\infty} t^{2} \ln \left(\frac{\left|m\left(t^{2}\right)+i t\right|^{2}}{4 \pi|t| \mu\left(t^{2}\right)}\right) d t=\frac{1}{8} \int_{0}^{\infty} q^{2}(x) d x
$$

if $q \in L^{2}(\mathbb{R})$, where

$$
\frac{\left|m\left(t^{2}\right)+i t\right|^{2}}{4 \pi|t| \mu\left(t^{2}\right)} \geq 1
$$

for a.e. $t$. Notice that since $\left|m\left(t^{2}\right)+i t\right|^{2} \geq t^{2}$, we have for any $I \subset \mathbb{R}^{+}$

$$
\int_{I} \ln \mu\left(t^{2}\right) d t>-C_{1}-C_{2} \int_{0}^{\infty} q^{2}(x) d x
$$


Definition 4. (Modified Blaschke product). If $\left\{-\xi_{j}^{2}\right\}$ are negative eigenvalues of $H_{l}$, then we define

$$
B_{m}(k)=\prod_{j}\left(\frac{k-i \xi_{j}}{k+i \xi_{j}} \exp \left(2 i k^{-1} \xi_{j}\right)\right)
$$

Lemma 5. For any $k \in \mathbb{C}^{+}$,

$$
a_{m}(k)=B_{m}(k) \exp \left(\frac{1}{2 \pi i} \int_{\mathbb{R}} K(t, k) \ln \left(\frac{\left|m\left(t^{2}\right)+i t\right|^{2}}{4 \pi|t| \mu\left(t^{2}\right)}\right) d t\right)
$$

Proof. Let us introduce the function

$$
h(k)=k^{2} \int_{\mathbb{R}} K(t, k) \ln \left(\frac{\left|m\left(t^{2}\right)+i t\right|^{2}}{4 \pi|t| \mu\left(t^{2}\right)}\right) d t=\int_{\mathbb{R}} \frac{t^{2}}{t-k} \ln \left(\frac{\left|m\left(t^{2}\right)+i t\right|^{2}}{4 \pi|t| \mu\left(t^{2}\right)}\right) d t
$$

This last integral is the Cauchy-type integral of a non-negative function from $L^{1}(\mathbb{R})$. For the truncated potential, we take

$$
f(k, R)=\int_{\mathbb{R}} \frac{t^{2}}{t-k} \ln \left(\frac{\left|m_{R}\left(t^{2}\right)+i t\right|^{2}}{4 \pi|t| \mu_{R}\left(t^{2}\right)}\right) d t=2 \pi i k^{2} \ln \left(a_{m}(k, R) B_{m}^{-1}(k, R)\right)
$$

For the imaginary parts of $f(k, R)$ and $h(k)$,

$$
\Im f(x+i y, R)=\int_{\mathbb{R}} \frac{y t^{2}}{(t-x)^{2}+y^{2}} \ln \left(\frac{\left|m_{R}\left(t^{2}\right)+i t\right|^{2}}{4 \pi|t| \mu_{R}\left(t^{2}\right)}\right) d t
$$

and

$$
\Im h(x+i y)=\int_{\mathbb{R}} \frac{y t^{2}}{(t-x)^{2}+y^{2}} \ln \left(\frac{\left|m\left(t^{2}\right)+i t\right|^{2}}{4 \pi|t| \mu\left(t^{2}\right)}\right) d t
$$

By repeating the proof of theorem 5.1 ([8], page 20), one has

$$
\Im h(k) \leq \liminf _{R \rightarrow \infty} \Im f(k, R), \quad k \in \mathbb{C}^{+}
$$

(This proof we refer to is based on several standard observations. We sketch the details here following [8]. If

$$
r_{R}(k)=\frac{i k-m_{R}\left(k^{2}\right)}{i k+m_{R}\left(k^{2}\right)}, \quad r(k)=\frac{i k-m\left(k^{2}\right)}{i k+m\left(k^{2}\right)}
$$

then $r_{R}(k) \rightarrow r(k)$ as $\Im k>0, \Re k>0$ and

$$
T(k)+|r(k)|^{2}=1, \quad T_{R}(k)+\left|r_{R}(k)\right|^{2}=1 \quad k \in \mathbb{R}
$$

where

$$
T(k)=\frac{4 k \pi \mu\left(k^{2}\right)}{\left|m\left(k^{2}\right)+i k\right|^{2}}, \quad T_{R}(k)=\frac{4 k \pi \mu_{R}\left(k^{2}\right)}{\left|m_{R}\left(k^{2}\right)+i k\right|^{2}}
$$


Since both $r_{R}(k)$ and $r(k)$ have nice boundary behavior near $\mathbb{R}^{+}$, one can prove $r_{R}(k) \rightarrow r(k)$ weakly in $L^{p}(a, b)$ for any $(a, b) \subset \mathbb{R}^{+}$and $p \in[1, \infty)$. Thus, for any $j=1,2 \ldots$

$$
\int_{a}^{b} \frac{y t^{2}}{(t-x)^{2}+y^{2}}|r(t)|^{2 j} d t \leq \liminf _{R} \int_{a}^{b} \frac{y t^{2}}{(t-x)^{2}+y^{2}}\left|r_{R}(t)\right|^{2 j} d t
$$

Using the Taylor expansion for $\ln \left(1-|r|^{2}\right)$, identities (2.22), and summing up the estimates above, we have

$$
\int_{a}^{b} \frac{y t^{2}}{(t-x)^{2}+y^{2}} \ln T(t) d t \geq \liminf _{R} \int_{a}^{b} \frac{y t^{2}}{(t-x)^{2}+y^{2}} \ln T_{R}(t) d t
$$

for any $a, b$. Taking $a \rightarrow 0, b \rightarrow \infty$ and handling $\mathbb{R}^{-}$similarly, we get (2.21).)

On the other hand,

$$
f(k, R) \rightarrow f(k)=2 \pi i k^{2} \ln \left(a_{m}(k) B_{m}^{-1}(k)\right)
$$

so

$$
0 \leq \Im h(k) \leq \Im f(k), \quad k \in \mathbb{C}^{+}
$$

Computing the asymptotics as $\Im k \rightarrow+\infty$, we have

$$
f(k) \sim-\frac{\pi}{4 k} \int_{0}^{\infty} q^{2}(x) d x+\frac{4 \pi}{3 k} \sum_{j} \xi_{j}^{3}
$$

by (2.23) and (2.15). Similarly,

$$
h(k) \sim-\frac{\pi}{4 k} \int_{0}^{\infty} q^{2}(x) d x+\frac{4 \pi}{3 k} \sum_{j} \xi_{j}^{3}
$$

Thus, by lemma 15 from Appendix, $f(k)=h(k)$ and the lemma easily follows.

As a simple corollary, we get

Lemma 6. For the modified Jost function,

$$
j_{m}(k)=\frac{2 i k B_{m}(k)}{m\left(k^{2}\right)+k i} \exp \left(\frac{1}{2 \pi i} \int_{\mathbb{R}} K(t, k) \ln \left(\frac{\left|m\left(t^{2}\right)+i t\right|^{2}}{4 \pi|t| \mu\left(t^{2}\right)}\right) d t\right), \quad k \in \mathbb{C}^{+}
$$

This formula is not the most natural one since the Blaschke product is built not from zeroes of $j_{m}$, those are hiding in the poles of $m\left(k^{2}\right)+i k$. Notice also that for a.e. $k \in \mathbb{R}$,

$$
\left|j_{m}(k)\right|^{2}=\frac{|k|}{\pi \mu\left(k^{2}\right)}
$$




\subsection{One result on weak convergence}

The main goal of this subsection is to prove

Theorem 7. As $R \rightarrow \infty$,

$$
\pi\left|j_{m}(k, R)\right|^{2} d \rho(E) \rightarrow \sqrt{E} d E
$$

in weak- $(*)$ topology on $\mathbb{R}^{+}$.

Proof. By the Spectral Theorem,

$$
\int_{\mathbb{R}} \frac{u(s, k) u(t, k)}{E-z} d \rho(E)=G(s, t, z), \quad z \in \mathbb{C}^{+}
$$

where $G(s, t, z)$ is the Green function for $H$ and the identity is understood in the distributional sense. Thus,

$$
I=\int_{\mathbb{R}} \frac{E u(s, k) u(t, k)+u^{\prime}(s, k) u^{\prime}(t, k)}{E-z} d \rho(E)=\delta(s-t)+z G(s, t, z)+\partial_{s t}^{2} G(s, t, z)
$$

Substituting the resolvent identity

$$
G=G_{0}-G_{0} * q * G
$$

and the explicit formula (2.11), we get (again, in the distributional sense)

$$
\begin{aligned}
& I=\sqrt{-z} e^{\sqrt{-z}|s-t|}-z\left(G_{0} * q * G\right)(s, t, z) \\
& +\left(\frac{\sqrt{-z}}{2}+\frac{1}{2 \sqrt{-z}}\right) e^{\sqrt{-z}(s+t)}+\left(\partial_{s}\left(G_{0} * q * G\right) \partial_{t}\right)(s, t, z)
\end{aligned}
$$

Taking imaginary part, we have for $z=x+i y$

$$
\begin{array}{r}
\int_{\mathbb{R}} \frac{y\left(E u(s, k) u(t, k)+u^{\prime}(s, k) u^{\prime}(t, k)\right)}{(E-x)^{2}+y^{2}} d \rho(E)= \\
=\Im\left(\sqrt{-z} e^{\sqrt{-z}|s-t|}-z\left(G_{0} * q * G\right)(s, t, z)\right. \\
\left.+\left(\frac{\sqrt{-z}}{2}+\frac{1}{2 \sqrt{-z}}\right) e^{\sqrt{-z}(s+t)}+\left(\partial_{s}\left(G_{0} * q * G\right) \partial_{t}\right)(s, t, z)\right)
\end{array}
$$

and the integral in the 1.h.s. converges for fixed $s$ and $t$ since

$$
\int \frac{d \rho(E)}{1+E^{2}}<\infty
$$

and $|u(s, k)|,|u(t, k)| \leq C k^{-1}$ as $k \rightarrow+\infty$ (by (2.6) and asymptotics of Jost functions). So, (2.26) holds as a functional identity. Take $s=t \rightarrow \infty$ and use $q \in L^{2}\left(\mathbb{R}^{+}\right)$to get

$$
\int_{\mathbb{R}} \frac{y\left(E u(s, k)^{2}+u^{\prime}(s, k)^{2}\right)}{(E-x)^{2}+y^{2}} d \rho(E)=\Im(\sqrt{-z})+\bar{o}(1), \quad s \rightarrow \infty
$$


In particular,

$$
\sup _{s>0} \int_{\mathbb{R}} \frac{E u(s, k)^{2}+u^{\prime}(s, k)^{2}}{E^{2}+1} d \rho(E)<\infty
$$

By the standard approximation argument, (2.7), and (2.28) we have

$$
\left|j_{m}(k, R)\right|^{2} d \rho(E) \rightarrow \pi^{-1} \sqrt{E} d E
$$

in the weak- $(*)$ topology on $(0, \infty)$.

\section{The asymptotics of modified Jost function on the real line}

In this section, we will prove

Theorem 8. For any finite interval I not containing zero,

$$
\begin{array}{r}
\int_{I}\left|\frac{j_{m}(k, R)}{j_{m}(k)}-1\right|^{2} d k \rightarrow 0, \\
\int_{I}\left|j_{m}(k, R)\right|^{2} d \rho_{s}(E) \rightarrow 0
\end{array}
$$

as $R \rightarrow \infty$.

Proof. The proof is standard. We will consider $I \subset \mathbb{R}^{+}$, the negative half-line can be handled similarly. Fix any $a, b$ such that $0<a<b$ and take isosceles triangle $T$ with base $[a, b]$ and the sides $\gamma_{1}, \gamma_{2}$. Denote the angle between $\gamma_{1(2)}$ and a base by $\phi$ and $\gamma=\gamma_{1} \cup \gamma_{2} \cup[a, b]$. Here, $\phi$ is a small positive angle to be chosen later. Take some reference interior point $\xi \in T$ and denote the harmonic measure by $\omega_{\xi}(t)$. We have two simple estimates

$$
\omega_{\xi}(t) \sim C|t-a|^{\pi / \phi-1}, \quad t \sim a, \quad \omega_{\xi}(t) \sim C|t-b|^{\pi / \phi-1}, \quad t \sim b
$$

The function $\omega_{\xi}(t)$ is nonnegative and smooth on the sides of triangle. Write

$$
0 \leq \int_{\gamma}\left|\frac{j_{m}(k, R)}{j_{m}(k)}-1\right|^{2} w_{\xi}(k) d|k|=\int_{\gamma}\left(\left|\frac{j_{m}(k, R)}{j_{m}(k)}\right|^{2}+1\right) w_{\xi}(k) d|k|-I
$$

and

$$
I=2 \Re \int_{\gamma} \frac{j_{m}(k, R)}{j_{m}(k)} w_{\xi}(k) d|k|=2 \Re \frac{j_{m}(\xi, R)}{j_{m}(\xi)} \rightarrow 2
$$

Consider

$$
A=\int_{\gamma_{1} \cup \gamma_{2}}\left|\frac{j_{m}(k, R)}{j_{m}(k)}\right|^{2} w_{\xi}(k) d|k|
$$

We need the following lemma 
Lemma 9. Consider $g(k, R)=j_{m}(k, R) / j_{m}(k)$ and fix any $a, b>0$. Then for $k \in \Omega=\{0<$ $\Im k<1, a<\Re k<b\}$ we have

$$
|g(k, R)|<C(\Im k)^{-1 / 2}
$$

uniformly in $R$.

Proof. The proof is elementary but lengthy. The multiplicative representation (2.24) yields

$$
g(k, R)=I_{1} I_{2} I_{3} I_{4}
$$

where

$$
\begin{gathered}
I_{1}=\frac{m\left(k^{2}\right)+i k}{m_{R}\left(k^{2}\right)+i k} \exp \left(\frac{1}{2 \pi i} \int_{a-\delta}^{b+\delta} \frac{t^{2}}{k^{2}(t-k)} \ln \left(\left|\frac{m_{R}\left(t^{2}\right)+i t}{m\left(t^{2}\right)+i t}\right|^{2}\right) d t\right) \\
I_{2}=\exp \left(\frac{1}{2 \pi i} \int_{a-\delta}^{b+\delta} \frac{t^{2}}{k^{2}(t-k)} \ln \left(\pi t^{-1}\left|j_{m}(t, R)\right|^{2} \mu\left(t^{2}\right)\right) d t\right) \\
I_{3}=\exp \left(\frac{1}{2 \pi i} \int_{t<a-\delta, t>b+\delta} \frac{t^{2}}{k^{2}(t-k)}\left[\ln \left(\frac{\left|m_{R}\left(t^{2}\right)+i t\right|^{2}}{4 \pi|t| \mu_{R}\left(t^{2}\right)}\right)-\ln \left(\frac{\left|m\left(t^{2}\right)+i t\right|^{2}}{4 \pi|t| \mu\left(t^{2}\right)}\right)\right] d t\right)
\end{gathered}
$$

and $\delta$ is positive small.

$$
I_{4}=B_{m}(k, R) B_{m}^{-1}(k)
$$

$I_{3}$ is uniformly bounded because

$$
t^{2} \ln \left(\frac{\left|m\left(t^{2}\right)+i t\right|^{2}}{4 \pi|t| \mu\left(t^{2}\right)}\right), t^{2} \ln \left(\frac{\left|m_{R}\left(t^{2}\right)+i t\right|^{2}}{4 \pi|t| \mu_{R}\left(t^{2}\right)}\right) \in L^{1}(\mathbb{R})
$$

with estimates uniform in $R$ by (2.18). $I_{4}$ is uniformly bounded since $\left\|\xi_{j}(R)\right\|_{\ell^{3}}$ is bounded uniformly in $R$ by (2.18) also.

$$
\left|I_{2}\right| \leq \exp \left(\Re \frac{1}{2 \pi i} \int_{a-\delta}^{b+\delta}\left(\frac{1}{(t-k)}+\frac{t+k}{k^{2}}\right) \ln \left(\pi t^{-1}\left|j_{m}(t, R)\right|^{2} \mu\left(t^{2}\right)\right) d t\right)
$$

We have

$$
\int_{a-\delta}^{b+\delta} \pi t^{-1}\left|j_{m}(t, R)\right|^{2} \mu\left(t^{2}\right) d t<C
$$

uniformly in $R$ by theorem 7 and

$$
\int_{0}^{\infty} \frac{t}{\pi\left|j_{m}(t, R)\right|^{2}\left(t^{4}+1\right)} d t=\int_{0}^{\infty} \frac{\mu_{R}\left(t^{2}\right)}{t^{4}+1} d t<C
$$

uniformly in $R$ by the standard estimates on the spectral measure. Moreover,

$$
\int_{a-\delta}^{b+\delta}\left|\ln \mu\left(t^{2}\right)\right| d t<C
$$


by (2.19). Therefore,

$$
\int_{a-\delta}^{b+\delta}\left|\frac{t+k}{k^{2}} \ln \left(\pi t^{-1}\left|j_{m}(t, R)\right|^{2} \mu\left(t^{2}\right)\right)\right| d t<C
$$

The application of Jensen's inequality and (3.6) to

$$
\exp \left(\Re \frac{1}{2 \pi i} \int_{a-\delta}^{b+\delta} \frac{1}{t-k} \ln \left(\pi t^{-1}\left|j_{m}(t, R)\right|^{2} \mu\left(t^{2}\right)\right) d t\right)
$$

gives

$$
\left|I_{2}\right|<C(\Im k)^{-1 / 2}
$$

(the standard estimate for the boundary growth of $H^{2}\left(\mathbb{C}^{+}\right)$functions). We are left with estimating $I_{1}$. Write

$$
I_{1}=\frac{P_{R}(k)}{P(k)}
$$

where

$$
P_{R}(k)=\frac{1}{m_{R}\left(k^{2}\right)+i k} \exp \left(\frac{1}{\pi i} \int_{a-\delta}^{b+\delta} \frac{t^{2}}{k^{2}(t-k)} \ln \left|m_{R}\left(t^{2}\right)+i t\right| d t\right)
$$

and

$$
P(k)=\frac{1}{m\left(k^{2}\right)+i k} \exp \left(\frac{1}{\pi i} \int_{a-\delta}^{b+\delta} \frac{t^{2}}{k^{2}(t-k)} \ln \left|m\left(t^{2}\right)+i t\right| d t\right)
$$

Let us show that

$$
0<C_{1}<|P(k)|<C_{2}, \quad 0<C_{1}<\left|P_{R}(k)\right|<C_{2}
$$

uniformly in $R$ and $k \in \Omega$. We will prove this for $P_{R}(k)$, the estimates for $P(k)$ are similar. Map $k \in\{\Im k>0, \Re k>0\}$ to $z \in \mathbb{C}^{+}$by $z=k^{2}$ and consider

$$
Q_{R}(z)=\frac{1}{m_{R}(z)+\sqrt{-z}} \exp \left(\frac{1}{\pi i} \int_{I} \frac{\sqrt{\xi}(\sqrt{\xi}+\sqrt{z})}{2 z} \frac{1}{\xi-z} \ln \left|m_{R}(\xi)+\sqrt{-\xi}\right| d \xi\right)
$$

where $I$ in the image of $[a-\delta, b+\delta]$. We have

$$
Q_{R}(z)=\frac{1}{m_{R}(z)+\sqrt{-z}} \exp \left(\frac{1}{\pi i} \int_{I} \frac{1}{\xi-z} \ln \left|m_{R}(\xi)+\sqrt{-\xi}\right| d \xi\right) M_{R}(z)
$$

with

$$
M_{R}(z)=\exp \left(\frac{1}{\pi i} \int_{I}\left(\frac{\sqrt{\xi}(\sqrt{\xi}+\sqrt{z})}{2 z}-1\right) \ln \left|m_{R}(\xi)+\sqrt{-\xi}\right| d \xi\right)
$$

Since

$$
0<C_{1}<\left|m_{R}(\xi)+\sqrt{-\xi}\right|<C_{2}\left|m_{R}(\xi)\right|+C_{3}
$$

and

$$
\int_{I}\left|m_{R}(\xi)\right|^{p} d \xi<C(p), \quad p<1
$$


uniformly in $R$ (by Kolmogorov's theorem), we have

$$
0<C_{1}<\left|M_{R}(z)\right|<C_{2}
$$

uniformly in $R$ and $z \in\left\{\Omega^{\prime}=\Omega^{2}\right\}$. The uniform in $R$ estimate

$$
0<C_{1}<\left|\frac{1}{m_{R}(z)+\sqrt{-z}} \exp \left(\frac{1}{\pi i} \int_{I} \frac{1}{\xi-z} \ln \left|m_{R}(\xi)+\sqrt{-\xi}\right| d t\right)\right|<C_{2}
$$

follows from lemma 16 in Appendix.

By (3.5), we have

$$
A \rightarrow \int_{\gamma_{1} \cup \gamma_{2}} w_{\xi}(k) d|k|, \quad R \rightarrow \infty
$$

provided that $\phi$ is taken small.

$$
\begin{gathered}
\int_{[a, b]}\left|\frac{j_{m}(k, R)}{j_{m}(k)}\right|^{2} w_{\xi}(k) d|k|+\pi / 2 \int_{\left[a^{2}, b^{2}\right]}\left|j_{m}(k, R)\right|^{2} w_{\xi}(k) k^{-2} d \rho_{s}(E)= \\
=\pi / 2 \int_{\left[a^{2}, b^{2}\right]}\left|j_{m}(k, R)\right|^{2} w_{\xi}(k) k^{-2} d \rho(E) \rightarrow \int_{[a, b]} w_{\xi}(k) d k
\end{gathered}
$$

by theorem 7. Combining (3.3), (3.4), (3.7), and (3.8), we get the statement of the theorem.

\section{Asymptotics of solutions to wave equation}

By the Spectral Theorem, we have the following representation for the solution to (1.1) with Cauchy data orthogonal to eigenstates corresponding to negative eigenvalues:

$$
y(x, t)=\int_{0}^{\infty} \cos (k t) u(x, k) \breve{\phi}(k) d \rho(E)+\int_{0}^{\infty} \frac{\sin (k t)}{k} u(x, k) \breve{\psi}(k) d \rho(E)
$$

where $\breve{\phi}, \breve{\psi}$ are generalized Fourier transforms of $\phi$ and $\psi$ associated with $H$. If one wants (4.1) to give the classical solution for $t>0$, it is sufficient to assume that, $\phi, \psi \in \mathcal{D}\left(\mathcal{H}^{\alpha}\right)$ for large $\alpha$ to guarantee the absolute convergence of the integrals after differentiation (e.g., $\alpha \geq 3 / 2$ is sufficient). We need to assume that $P_{(-\infty, 0)} \phi=P_{(-\infty, 0)} \psi=0$ since otherwise the solutions can grow exponentially and thus are not physical.

Consider the second integral in (4.1). We assume

$$
\breve{\psi}(k) / k \in L^{2}(d \rho(k))
$$


which is equivalent to $\psi=\sqrt{|H|} g$ for some $g$. This assumption is rather natural. Indeed, if $H$ has eigenvalue at zero with eigenfunction $\phi$, then its contribution will yield a linear growth

$$
\|u(x, t)\|_{2} \geq C t
$$

This will occur even in the free case when $\psi$ does not oscillate. Although some analysis is possible in the general case, we will work with stable situation when the $L^{2}\left(\mathbb{R}^{+}\right)$norm of the solution is bounded in time and so will require (4.2). We prove the pointwise "large time - large coordinate" translation-like asymptotics for the initial data in the set dense in $\sigma_{\mathrm{ac}}(H)$. For large $t$ and small $x$, the solution can behave in rather chaotic way due to the possible presence of embedded singular continuous spectrum.

Theorem 10. Assume that $q \in L^{2}\left(\mathbb{R}^{+}\right)$and

$$
\int_{0}^{\infty} q(x) d x
$$

converges. Take $f \in \mathcal{D}(\sqrt{|H|}), P_{(-\infty, 0)} f=0$ and let $\phi=f$ and $\psi=-i \sqrt{|H|} f$. Then, for any fixed $x$, we have

$$
y(T+x, T) \rightarrow \mu_{f}(x), \quad T \rightarrow \infty
$$

where $\mu_{f}(x) \in W^{1,2}(\mathbb{R})$.

Proof. Clearly, for this choice of initial conditions,

$$
y(x, t)=e^{-i t \sqrt{|H|}} f
$$

and we need to control $e^{-i T \sqrt{|H|}} f$ suitably scaled. If $H$ has zero eigenvalue with eigenfunction $e_{0}(x)$, then the solution of wave equation with initial condition $\left\{e_{0}(x), 0\right\}$ is stationary, i.e. $y_{0}(x, t)=e_{0}(x) \rightarrow 0$ as $x \rightarrow \infty$ so we can always assume without loss of generality that $f$ is orthogonal to $e_{0}$. We have

$$
\int\left(k^{2}+1\right)|\breve{f}(k)|^{2} d \rho(E)<\infty
$$

which guarantees the absolute convergence of the integrals in (4.1) due to (2.28).

Take any $\delta, M, 0<\delta<M$. Then

$$
y(T+x, T)=I_{1}+I_{2}+I_{3}+I_{4}
$$

where

$$
\begin{gathered}
I_{1}=\int_{\delta}^{M} e^{-i k T} \breve{f}(k) u(T+x, k) d \rho_{s}(E) \\
I_{2}=\int_{\delta}^{M} e^{-i k T} \breve{f}(k) u(T+x, k) \mu\left(k^{2}\right) d E
\end{gathered}
$$




$$
\begin{aligned}
I_{3} & =\int_{0}^{\delta} e^{-i k T} \breve{f}(k) u(T+x, k) d \rho(E) \\
I_{4} & =\int_{M}^{\infty} e^{-i k T} \breve{f}(k) u(T+x, k) d \rho(E)
\end{aligned}
$$

By (2.6), (3.2), and (4.3) $I_{1}$ converges to zero for $T \rightarrow \infty$. Consider $I_{3}$. Let

$$
g(x)=\int_{0}^{\delta} e^{-i k T} \breve{f}(k) u(x, k) d \rho(E)
$$

By the Spectral Theorem, we have $\|g\|_{2} \rightarrow 0,\left\|g^{\prime}\right\|_{2} \rightarrow 0$ as $\delta \rightarrow 0$ and so $\|g\|_{\infty} \rightarrow 0$ as $\delta \rightarrow 0$. For $I_{4}$, Cauchy-Schwarz yields

$$
\sup _{T, x}\left|I_{4}\right| \leq \sup _{T, x}\left(\int_{M}^{\infty}|\breve{f}(k)|^{2}\left(k^{2}+1\right) d \rho(E)\right)^{1 / 2}\left(\int_{M}^{\infty} \frac{u(T+x, k)^{2}}{E+1} d \rho(E)\right)^{1 / 2} \rightarrow 0,
$$

as $M \rightarrow \infty$ by (2.28) and (4.3). For $I_{2}$, we have $\left(\delta_{1}=\sqrt{\delta}, M_{1}=\sqrt{M}\right)$

$$
\begin{array}{r}
I_{2}=\frac{1}{\pi i} \int_{\delta_{1}}^{M_{1}} e^{-i k T} \breve{f}(k)\left(\overline{j_{m}(T+x, k)} e^{i k(T+x)-i \phi(0, k, T+x)}\right. \\
\left.-j_{m}(T+x, k) e^{-i k(T+x)+i \phi(0, k, T+x)}\right) \frac{k d k}{\left|j_{m}(k)\right|^{2}}
\end{array}
$$

where we used (2.25) and (2.6). By (4.3),

$$
\breve{f}(k)=j_{m}(k) h(k) k^{-1}
$$

and $\left(k^{2}+1\right)^{1 / 2} h(k) \in L^{2}\left(\mathbb{R}^{+}\right)$. For any $\delta_{1}, M_{1}>0$,

$$
\begin{array}{r}
\int_{\delta_{1}}^{M_{1}} e^{-i k T} h(k)\left(\frac{\overline{j_{m}(T+x, k)}}{\overline{j_{m}(k)}} e^{i k(T+x)-i \phi(0, k, T+x)}\right. \\
\left.-\frac{j_{m}(T+x, k)}{j_{m}(k)} \frac{\bar{j}_{m}(k)}{\overline{j_{m}(k)}} e^{-i k(T+x)+i \phi(0, k, T+x)}\right) d k
\end{array}
$$

converges to

$$
\exp \left(-\frac{i}{2 k} \int_{0}^{\infty} q(x) d x\right) \int_{\delta_{1}}^{M_{1}} h(k) e^{i k x} d k
$$

by (3.1) and Riemann-Lebesgue lemma. Since $M_{1}$ and $\delta_{1}$ are arbitrary and $h$ decays fast, the theorem is true with

$$
\mu_{f}(x)=\frac{1}{\pi i}\left(\int_{0}^{\infty} h(k) e^{i k x} d k\right) \exp \left(-\frac{i}{2 k} \int_{0}^{\infty} q(x) d x\right)
$$


Taking the conjugation, one can prove a similar result for initial conditions of the form $\{f, i \sqrt{|H|} f\}$. Since

$$
\{f, \sqrt{|H|} g\}=\left\{f_{1}, i \sqrt{|H|} f_{1}\right\}+\left\{f_{2},-i \sqrt{|H|} f_{2}\right\}
$$

with $f_{1}=(f-i g) / 2 f_{2}=(f+i g) / 2$, the theorem holds in general case. Clearly, the simplest way to satisfy $\psi=\sqrt{|H|} g$ with some $g$ is to take $\psi=0$.

Remark. How should one take a function $f$ to guarantee that at least part of the wave will travel ballistically after taking $P_{[0, \infty)} f$ as initial value in Cauchy problem? The theorem just proved tells us that $f$ should have some nontrivial part in a.c. subspace. Checking that is not easy in general but if one takes $f$ to be compactly supported, then $d\left(P_{E} f, f\right) / d E>0$ for a.e. $E>0$. The latter statement can be easily proved by adjusting technique from [6]. The theorem 10 requires the convergence of the integral

$$
\int_{0}^{\infty} q(x) d x
$$

That can not be achieved for $q \notin L^{1}\left(\mathbb{R}^{+}\right)$unless $q$ oscillates but if it does, then the negative spectrum might easily appear and therefore we have to project away from the negative eigenspace which is not an easy thing to do (though possible of course by Gram-Schmidt process). In the next section, we will not require (4.4) and so the asymptotics will be established for more tangible set of initial data.

\section{Modified wave operators}

In the previous section, we assumed the conditional convergence to prove that the a.c. part of the wave undergoes the simple translation. If the potential is only square summable, this is not the case anymore. We will study the long-time dynamics by considering the modified wave operators. Notice that if $H \geq 0$, then the group $e^{i t \sqrt{H}}$ gives the formal solution to the wave equation.

Let us start with some notations. Take any function $f(x) \in L^{2}\left(\mathbb{R}^{+}\right)$. Then

$$
\begin{aligned}
& e^{i t \sqrt{H_{0}}} f=\frac{2}{\pi} \int_{0}^{\infty} e^{i k t} \sin (k x) \tilde{f}(k) d k= \\
& =\frac{1}{2 \pi}\left(\int_{0}^{\infty} e^{i k(t-x)} \hat{f}_{o}(k) d k-\int_{0}^{\infty} e^{i k(t+x)} \hat{f}_{o}(k) d k\right)
\end{aligned}
$$

where

$$
\tilde{f}(k)=\int_{0}^{\infty} f(x) \sin (k x) d x=-i \hat{f}_{o}(k) / 2
$$

$f_{o}(x)$ is the odd continuation of $f(x)$ and $\hat{f}_{o}(k)$ is its (inverse) Fourier transform. Thus, for $t \rightarrow$ $+\infty$

$$
e^{i t \sqrt{H_{0}}} f \sim \frac{1}{2 \pi} \int_{0}^{\infty} e^{i k(t-x)} \hat{f}_{o}(k) d k
$$


in $L^{2}\left(\mathbb{R}^{+}\right)$. Consider the multiplier

$$
M(t, k)=\exp \left(i k t+\frac{i}{2 k} \int_{0}^{t} q(s) d s\right)
$$

and

$$
[W(t) f](x)=\frac{1}{2 \pi} \int_{0}^{\infty} e^{-i k x} M(k, t) \hat{f}_{o}(k) d k
$$

The main result of this section is

Theorem 11. Let $q \in L^{2}\left(\mathbb{R}^{+}\right)$and

$$
|q(x)|<C(1+x)^{-1 / 2}
$$

If $H_{1}=P_{[0, \infty)} H$, then the following strong limit exists

$$
(\mathrm{s})-\lim _{t \rightarrow+\infty} e^{-i t \sqrt{H_{1}}} W(t)
$$

in $L^{2}\left(\mathbb{R}^{+}\right)$norm.

Proof. Since $W(t)$ is unitary, it is sufficient to prove existence of the limit for functions $f(x)$ with $\hat{f}_{o}(k)$ being infinitely smooth and compactly supported on, say, $[a, b] \subset \mathbb{R}^{+}$. Let

$$
\omega(t)=\left|\int_{0}^{t}\right| q(x)|d x / \sqrt{t}|
$$

Obviously, $\omega(t) \rightarrow 0$ as $t \rightarrow \infty$ but $\omega(t)>C t^{-1 / 2}$ unless $q=0$.

Lemma 12. Consider

$$
g_{t}(x)=\int_{0}^{\infty} \exp \left(-i k x+\frac{i}{2 k} \int_{0}^{t} q(s) d s\right) \hat{f}_{o}(k) d k
$$

Then,

$$
\int_{|x|>\sqrt{t \omega(t)}}\left|g_{t}(x)\right|^{2} d x \rightarrow 0, \quad t \rightarrow \infty
$$

Proof. We have

$$
\int x^{2}\left|g_{t}(x)\right|^{2} d x \lesssim \int\left|\hat{f}_{o}^{\prime}(k)\right|^{2} d k+\left(\int_{0}^{t}|q(s)| d s\right)^{2} \int k^{-2}\left|\hat{f}_{o}(k)\right|^{2} d k \lesssim 1+t \omega^{2}(t)
$$

which proves the claim.

Thus, the lemma says that $W(t) f$ is localized on $|x-t|<\sqrt{t \omega(t)}$ in $L^{2}$ sense. We also need the following lemma 
Lemma 13. We have

$$
\sup _{\alpha, \beta, k, t}\left|\int_{\alpha}^{\beta} e^{i x k}[W(t) f](x) d x\right|<\infty
$$

Proof. It is sufficient to prove

$$
\sup _{\alpha, \beta, k, T_{1}}\left|\int_{0}^{\infty} \frac{e^{i(\xi-k) \beta}-e^{i(\xi-k) \alpha}}{\xi-k} \exp \left(\frac{i T_{1}}{\xi}\right) \hat{f}_{o}(\xi) d \xi\right|<\infty
$$

Since $\hat{f}_{o}$ is infinitely smooth with support on $[a, b] \subset(0, \infty)$, we just need to show that, say,

$$
\sup _{\gamma, T_{1}}\left|\int_{1 / 2}^{2} \frac{e^{i(\xi-1) \gamma}}{\xi-1} \exp \left(\frac{i T_{1}}{\xi}\right) d \xi\right|<\infty
$$

where integral is understood in v.p. sense. This is proved in lemma 17 in Appendix.

Denote the generalized Fourier transform of $d_{t}(x)=\chi_{|x-t|<\sqrt{t \omega(t)}} W(t) f$ by $\breve{d}_{t}(k)$. We have

$$
\begin{gathered}
\breve{d}_{t}(k)=\frac{1}{2 i k} \int_{t-\sqrt{t \omega(t)}}^{t+\sqrt{t \omega(t)}}\left(\overline{j_{m}(k, x)} e^{i x k-i \phi(0, k, x)}-j_{m}(k, x) e^{-i x k+i \phi(0, k, x)}\right)[W(t) f](x) d x \\
=I_{1}-I_{2}
\end{gathered}
$$

where

$$
I_{1}=\frac{1}{2 i k} \int_{t-\sqrt{t \omega(t)}}^{t+\sqrt{t \omega(t)}} \overline{j_{m}(k, x)} e^{i x k-i \phi(0, k, x)}[W(t) f](x) d x
$$

Integrating by parts,

$$
\begin{gathered}
I_{1}=\frac{1}{2 i k} \overline{j_{m}}(k, t-\sqrt{t \omega(t)}) \exp (-i \phi(0, k, t-\sqrt{t \omega(t)})) \int_{t-\sqrt{t \omega(t)}}^{t+\sqrt{t \omega(t)}} e^{i x k}[W(t) f](x) d x+J_{1} \\
J_{1}=\frac{1}{2 i k} \int_{t-\sqrt{t \omega(t)}}^{t+\sqrt{t \omega(t)}}\left(\overline{j_{m}(k, x)} \exp (-i \phi(0, k, x))\right)_{x}^{\prime}\left(\int_{x}^{t+\sqrt{t \omega(t)}} e^{i k u}[W(t) f](u) d u\right) d x
\end{gathered}
$$

By (2.8) and the lemma 13,

$$
\left|J_{1}\right| \lesssim \int_{t-\sqrt{t \omega(t)}}^{t+\sqrt{t \omega(t)}}|q(x)|\left|j_{m}(k, x)\right| d x
$$

for any $k \in I \subset \mathbb{R}^{+}$. So, by Minkowski and (2.28),

$$
\left(\int_{I}\left|J_{1}\right|^{2} d \rho(E)\right)^{1 / 2} \lesssim \int_{t-\sqrt{t \omega(t)}}^{t+\sqrt{t \omega(t)}}|q(x)|\left(\int_{I}\left|j_{m}(k, x)\right|^{2} d \rho(E)\right)^{1 / 2} d x
$$




$$
<C \sqrt{\omega(t)} \rightarrow 0
$$

The first term in (5.3) can be written as

$$
\begin{gathered}
K_{1}=\frac{1}{2 i k} \overline{j_{m}}(k, t-\sqrt{t \omega(t)}) \hat{f}_{o}(k) \exp \left(i k t+\frac{i}{2 k} \int_{t-\sqrt{t \omega(t)}}^{t} q(u) d u\right) \\
+\frac{1}{2 i k} \overline{j_{m}}(k, t-\sqrt{t \omega(t)}) \exp (-i \phi(0, k, t-\sqrt{t \omega(t)})) \int_{|x-t|>\sqrt{t \omega(t)}} e^{i x k}[W(t) f](x) d x
\end{gathered}
$$

The second term converges to zero in $L^{2}\left(I, d \rho_{s}(E)\right)$ because

$$
\int_{|x-t|>\sqrt{t \omega(t)}} e^{i x k}[W(t) f](x) d x
$$

is uniformly bounded by lemma 13 and

$$
\int_{I}\left|j_{m}(k, t)\right|^{2} d \rho_{s}(E) \rightarrow 0, \quad t \rightarrow \infty
$$

by (3.2). It also converges to zero in $L^{2}\left(I, \mu\left(k^{2}\right) d E\right)$ by (3.1) and since

$$
\left\|\int_{|x-t|>\sqrt{t \omega(t)}} e^{i x k}[W(t) f](x) d x\right\|_{L^{2}(d k, \mathbb{R})} \rightarrow 0, \quad t \rightarrow \infty
$$

by (5.2). Therefore, by (3.1), we again have

$$
e^{-i k t} K_{1} \rightarrow \frac{1}{2 i k} \overline{j_{m}}(k) \hat{f}_{o}(k) \chi_{\Theta}(E), \quad t \rightarrow \infty
$$

in $L^{2}(I, d \rho(E))$ where $\Theta$ is the complement of the support of $d \rho_{s}(E)$ and $I \subset(0, \infty)$. The estimates for the $I_{2}$ are similar. They yield

$$
\int_{I}\left|I_{2}\right|^{2} d \rho(E) \rightarrow 0, t \rightarrow \infty
$$

Thus, we have

$$
\int_{I}\left|\breve{d}_{t}(k) e^{-i k t}-\frac{1}{2 i k} \overline{j_{m}}(k) \hat{f}_{o}(k) \chi_{\Theta}(E)\right|^{2} d \rho(E) \rightarrow 0, \quad t \rightarrow \infty
$$

for any $I \subset \mathbb{R}^{+}$. If the generalized Fourier transform of $w_{t}(x)=W(t) f$ is denoted by $\breve{w}_{t}(k)$, then

$$
\int_{I}\left|\breve{w}_{t}(k) e^{-i k t}-\frac{1}{2 i k} \overline{j_{m}}(k) \hat{f}_{o}(k) \chi_{\Theta}(E)\right|^{2} d \rho(E) \rightarrow 0, \quad t \rightarrow \infty
$$


for any interval $I=[\delta, M]$. On the other hand, notice that $w_{t}(x) \in W^{l, 2}(\mathbb{R})$ for any $l \geq 0$, $\sup _{t>0}\left\|\partial_{x x}^{2} w_{t}(x)\right\|_{2}<\infty$, and $w_{t}(x)$ is concentrated around $t$ by lemma 12. If $p(x)$ is infinitely smooth, $p(x)=0$ for $x<0$ and $p(x)=1$ for $x>1$, then $p(x) w_{t}(x) \in \mathcal{D}(\mathcal{H})$ and $\sup _{t}\left\|H\left(p(x) w_{t}(x)\right)\right\|_{2}<\infty$. Therefore,

$$
\limsup _{t \rightarrow \infty} \int_{M}^{\infty}\left\|\breve{w}_{t}(k)\right\|_{2}^{2} d \rho(E) \rightarrow 0, \quad M \rightarrow \infty
$$

and

$$
\int_{\delta}^{\infty}\left|\breve{w}_{t}(k) e^{-i k t}-\frac{1}{2 i k} \overline{j_{m}}(k) \hat{f}_{o}(k) \chi_{\Theta}(E)\right|^{2} d \rho(E) \rightarrow 0
$$

for any $\delta>0$. Let us now show that no $L^{2}(d \rho(E))$ norm of $\breve{w}_{t}(k)$ can concentrate around zero energy. In other words, that

$$
\limsup _{t \rightarrow \infty}\left\|P_{[0, \delta]} W(t) f\right\| \rightarrow 0
$$

as $\delta \rightarrow 0$.

By definition of $W(t)$, we have

$$
W(t) f=v_{t}^{\prime \prime}(x-t)
$$

where $v_{t} \in W^{2,2}(\mathbb{R})$ and its $L^{2}$ norm is concentrated on $[-\sqrt{t}, \sqrt{t}]$. Thus

$$
W(t) f=H s_{1}(t)+s_{2}(t)
$$

where $s_{1}(t) \in \mathcal{D}(\mathcal{H})$ and

$$
\sup _{t>0}\left(\left\|s_{1}(t)\right\|_{2}+\left\|H s_{1}(t)\right\|_{2}\right)<\infty
$$

For $s_{2}$,

$$
\left\|s_{2}\right\|_{L^{2}\left(\mathbb{R}^{+}\right)} \rightarrow 0, \quad t \rightarrow \infty
$$

Therefore,

$$
\int_{0}^{\delta}\left|\breve{w}_{t}(k)\right|^{2} d \rho(E) \lesssim \int_{0}^{\delta}\left(E\left|\breve{s}_{1}(k)\right|^{2}+\left|\breve{s}_{2}(k)\right|^{2}\right) d \rho(E) \lesssim \delta+\bar{o}(1), \quad t \rightarrow \infty
$$

Therefore, we have (5.5) and so

$$
\int_{0}^{\infty}\left|\breve{w}_{t}(k) e^{-i k t}-\frac{1}{2 i k} \overline{j_{m}}(k) \hat{f}_{o}(k) \chi_{\Theta}(E)\right|^{2} d \rho(E) \rightarrow 0
$$

which proves the theorem. 
If the potential is nonnegative then $H \geq 0$ and we do not have to project to the positive part of the spectrum. We were working with the group $e^{i t \sqrt{H_{1}}}$ to guarantee stability in $L^{2}\left(\mathbb{R}^{+}\right)$, after all we established asymptotics in this norm.

Using simple contour integration technique, one can show that

$$
\sup _{|x|<T} \int_{0}^{\infty} \exp \left(-i k x+\frac{i T_{1}}{k}\right) \hat{f}_{o}(k) d k \rightarrow 0
$$

as $T_{1} \rightarrow \infty$ provided that infinitely smooth function $f_{o}$ has compact support in $(0, \infty)$ (in fact, the bound is $\sim T_{1}^{-1 / 2}$ ). That shows that the $L^{2}$ norm of $W(t) f$ might be smeared over the interval $[t-\sqrt{t}, t+\sqrt{t}]$ and the strength of smearing depends on the size of

$$
\int_{0}^{T} q(x) d x
$$

This required us to make an additional assumption (5.1). In the meantime, some results can be proved even without (5.1). For example, the methods presented in [3], allow us to prove

Proposition 14. Let $q \in L^{2}\left(\mathbb{R}^{+}\right)$. If $H_{1}=P_{[0, \infty)} H$, then for any $f \in L^{2}\left(\mathbb{R}^{+}\right)$

$$
\frac{1}{T} \int_{0}^{T}\left\|e^{-i t \sqrt{H_{1}}} W(t) f-G(f)\right\|_{2}^{2} d t \rightarrow 0
$$

The formula for $G(f)$ can be given explicitly in terms of generalized Fourier transform. (We had to use (5.1) at (5.4). In the meantime, we have

$$
\begin{array}{r}
\frac{1}{T} \int_{0}^{T}\left(\int_{t-\sqrt{t}}^{t+\sqrt{t}}|q(x)| d x\right)^{2} d t \lesssim \frac{1}{T} \int_{0}^{T} \sqrt{t} \int_{t-\sqrt{t}}^{t+\sqrt{t}} q^{2}(x) d x d t \\
\lesssim \frac{1}{T} \int_{0}^{C T}(1+x) q^{2}(x) \rightarrow 0, \quad T \rightarrow \infty
\end{array}
$$

assuming only $q \in L^{2}\left(\mathbb{R}^{+}\right)$. That leads to the proof of proposition.)

The existence of wave operators in the form we proved also establishes the long-time asymptotics for the initial data from the a.c. part of the spectrum. If $f$, the initial value for $e^{i t \sqrt{H}}$ is compactly supported, then its spectral measure has a.c. part supported on $\mathbb{R}^{+}$. If $f_{1}=P_{\mathrm{ac}} f, f_{2}=P_{\mathrm{s}} f$, and $y_{1}(t)=\exp (i t \sqrt{H}) f_{1}, y_{2}(t)=\exp (i t \sqrt{H}) f_{2}$, then $y_{1} \perp y_{2}$ for any $t$. We do not know whether $y_{2}$ travels ballistically in $L^{2}$ norm, but since $y_{1}$ does (for most time if $q \in L^{2}\left(\mathbb{R}^{+}\right)$and for all times if we additionally assume (5.1)), the whole wave $y=y_{1}+y_{2}$ must travel ballistically too. Indeed,

$$
\begin{array}{r}
\int_{t-\sqrt{t}}^{t+\sqrt{t}}|y(x, t)|^{2} d x=\int_{t-\sqrt{t}}^{t+\sqrt{t}}\left|y_{1}(x, t)\right|^{2} d x+\int_{t-\sqrt{t}}^{t+\sqrt{t}}\left|y_{2}(x, t)\right|^{2} d x \\
+2 \Re \int_{t-\sqrt{t}}^{t+\sqrt{t}} y_{1}(x, t) \overline{y_{2}(x, t)} d x
\end{array}
$$


and since

$$
\int_{t-\sqrt{t}}^{t+\sqrt{t}} y_{1}(x, t) \overline{y_{2}(x, t)} d x \rightarrow 0
$$

by orthogonality and localization of $y_{1}$, we have that

$$
\limsup _{t \rightarrow \infty} \int_{t-\sqrt{t}}^{t+\sqrt{t}}|y(x, t)|^{2} d x \geq\left\|f_{1}\right\|_{2}^{2}
$$

if $q$ satisfies conditions of theorem 11. If $q$ is only square summable, we still have ballistic propagation for some part of the wave for most of the time. Notice also that the speed of propagation is always finite, i.e. if the initial data is compactly supported on $(0, a)$ then $y(x, t)=0$ for $x>a+t$. Thus the motion can not be faster than ballistic. The finite speed of propagation follows, e.g., from the Duhamel expansion.

As was mentioned earlier, for any $p>2$ there is some $q \in L^{p}\left(\mathbb{R}^{+}\right)$such that the spectrum is pure point. This, of course, leads to localization of the wave. Indeed, for any $f$,

$$
P_{[0, \infty)} f=\sum_{j}\left\langle f, e_{j}\right\rangle e_{j}
$$

where $e_{j}$ are $L^{2}\left(\mathbb{R}^{+}\right)$-normalized eigenfunctions corresponding to nonnegative eigenvalues $\lambda_{j}$. Therefore,

$$
e^{i t \sqrt{H_{1}}} P_{[0, \infty)} f=\sum_{j=1}^{N} e^{i \sqrt{\lambda_{j}} t}\left\langle f, e_{j}\right\rangle e_{j}+\epsilon_{N}(t)
$$

where $\sup _{t}\left\|\epsilon_{N}(t)\right\|_{2} \rightarrow 0$ as $N \rightarrow \infty$. The first term, though, is localized around the origin in $L^{2}\left(\mathbb{R}^{+}\right)$for any time so we have localization of the whole wave.

\section{Appendix}

In this appendix, we collected some simple results we used in the main text.

Lemma 15. Assume that $g(k)$ is positive harmonic function in $\mathbb{C}^{+}$and

$$
y g(i y) \rightarrow 0
$$

as $y \rightarrow+\infty$. Then, $g(k)=0$

Proof. We have integral representation for $g$

$$
g(i y)=\beta y+\int_{\mathbb{R}} \frac{y}{y^{2}+t^{2}} d \tau(t)
$$


where $\beta \geq 0$ and for the positive measure $d \tau$

$$
\int_{\mathbb{R}} \frac{d \tau(t)}{1+t^{2}}<\infty
$$

Multiply the both sides of (6.1) by $y$ and take $y \rightarrow \infty$. Then, $\beta=0$ and $d \tau=0$ so we get the statement of the lemma.

Lemma 16. Assume that $f(z)$ is Herglotz function in $\mathbb{C}^{+}$. Take any interval $[a, b]$ and $\delta>0$. Then,

$$
0<C_{1}<\left|\frac{1}{f(z)} \exp \left(\frac{1}{\pi i} \int_{a-\delta}^{b+\delta} \frac{1}{\xi-z} \ln |f(\xi)| d \xi\right)\right|<C_{2}
$$

uniformly in $\Omega_{1}=\{0<\Im z<1, a<\Re z<b\}$. The constants $C_{1(2)}$ depend only on $|f(i)|$.

Proof. Map $\mathbb{C}^{+}$to $\mathbb{D}$ by $k(z)=(z-i)(z+i)^{-1}$. If $z(k)=i(k+1)(1-k)^{-1}$ and $F(k)=f(z(k))$, then (6.2) is equivalent to proving

$$
0<C_{1}<\left|\frac{1}{F(k)} \exp \left(\frac{1}{\pi i} \int_{\theta_{1}-\delta_{1}}^{\theta_{2}+\delta_{2}} \frac{1}{z(u)-z(k)} \ln |F(u)| d z(u)\right)\right|<C_{2}
$$

in the subset $\Omega_{2}=k\left(\Omega_{1}\right)$ of $\mathbb{D}$ adjacent to the arc $k([a, b])=\left(e^{i \theta_{1}}, e^{i \theta_{2}}\right)$. Since $F$ is outer, we have

$$
F(k)=\exp \left(\frac{1}{2 \pi} \int_{-\pi}^{\pi} \frac{e^{i \theta}+k}{e^{i \theta}-k} \ln \left|F\left(e^{i \theta}\right)\right| d \theta\right)
$$

After substitution, it is sufficient to show that

$$
\begin{gathered}
\left|\int_{\theta_{1}-\delta_{1}}^{\theta_{2}+\delta_{1}}\left(\frac{(1-k) e^{i \theta}}{\pi\left(1-e^{i \theta}\right)\left(e^{i \theta}-k\right)}-\frac{e^{i \theta}+k}{2 \pi\left(e^{i \theta}-k\right)}\right) \ln \right| F\left(e^{i \theta}\right)|d \theta|<C \\
\left|\int_{\mathbb{T} \backslash\left[\theta_{1}-\delta_{1}, \theta_{2}+\delta_{1}\right]} \frac{e^{i \theta}+k}{e^{i \theta}-k} \ln \right| F\left(e^{i \theta}\right)|d \theta|<C
\end{gathered}
$$

Notice that we have

$$
\int_{\mathbb{T}}\left|F\left(e^{i \theta}\right)\right|^{p} d \theta<C(p,|F(0)|), \quad p<1
$$

by Kolmogorov's theorem and since $\ln \left|F\left(e^{i \theta}\right)\right| \in L^{1}(\mathbb{T})$. These estimates prove (6.3) and (6.4) in $\Omega_{2}$.

Lemma 17. The following estimate holds

$$
\sup _{\gamma, T_{1}}\left|\int_{1 / 2}^{2} \frac{e^{i(\xi-1) \gamma}}{\xi-1} \exp \left(\frac{i T_{1}}{\xi}\right) d \xi\right|<\infty
$$

where integral is understood in v.p. sense. 
Proof. Interesting cases are when $\left|T_{1}\right|$ or $|\gamma|$ are large. Making the substitution $\gamma(\xi-1)=\xi_{1}$ and absorbing the constants, we need to show

$$
\sup _{\gamma, T_{2}}\left|\int_{-\gamma / 2}^{\gamma} \frac{e^{i \xi_{1}}}{\xi_{1}} \exp \left(\frac{i T_{2}}{\xi_{1}+\gamma}\right) d \xi_{1}\right|<C
$$

or, essentially,

$$
\sup _{\gamma, T_{2}}\left|\int_{-\gamma / 2}^{\gamma / 2} \frac{e^{i \xi_{1}}}{\xi_{1}} \exp \left(\frac{i T_{2}}{\xi_{1}+\gamma}\right) d \xi_{1}\right|<C
$$

Let $\xi_{2}=\xi_{1}+\gamma$, then

$$
\sup _{\gamma, T_{2}}\left|\int_{\gamma / 2}^{3 \gamma / 2} \frac{\exp \left(i\left(\xi_{2}+\frac{T_{2}}{\xi_{2}}\right)\right)}{\xi_{2}-\gamma} d \xi_{2}\right|=\sup _{a, T}\left|\int_{a}^{3 a} \frac{\exp \left(i T\left(\xi \pm \xi^{-1}\right)\right)}{\xi-2 a} d \xi\right|
$$

We can always assume that $a, T>0$. Consider the first case, i.e.

$$
\sup _{a, T}\left|\int_{a}^{3 a} \frac{\exp \left(i T\left(\xi-\xi^{-1}\right)\right)}{\xi-2 a} d \xi\right|
$$

The boundedness of the last expression can be proved by contour integration. Indeed, take the contour $\Gamma_{a, \delta}=\gamma_{1} \cup \gamma_{2} \cup \gamma_{3}$, where

$$
\begin{gathered}
\gamma_{1}=\{\delta<|\xi-2 a|<a, \quad \Im \xi=0\} \\
\gamma_{2}=\{\Im \xi \geq 0, \quad|\xi-2 a|=a\} \\
\gamma_{3}=\{\Im \xi \geq 0, \quad|\xi-2 a|=\delta\}
\end{gathered}
$$

Notice that $\Im\left(\xi-\xi^{-1}\right) \geq 0$ on $\Gamma_{a, \delta}$. Therefore,

$$
\left|\int_{a}^{3 a} \frac{\exp \left(i T\left(\xi-\xi^{-1}\right)\right)}{\xi-2 a} d \xi\right|=\lim _{\delta \rightarrow 0}\left|\int_{\gamma_{2} \cup \gamma_{3}} \frac{\exp \left(i T\left(\xi-\xi^{-1}\right)\right)}{\xi-2 a} d \xi\right|<C
$$

with constant $C$ independent of $a$ and $T$. Consider the second case,

$$
\sup _{a, T}\left|\int_{a}^{3 a} \frac{\exp \left(i T\left(\xi+\xi^{-1}\right)\right)}{\xi-2 a} d \xi\right|
$$

If $\Im \xi, \Re \xi>0$ and $|\xi|>1$, then the same contour integration gives a necessary estimate. Thus,

$$
\sup _{a>1, T>0}\left|\int_{a}^{3 a} \frac{\exp \left(i T\left(\xi+\xi^{-1}\right)\right)}{\xi-2 a} d \xi\right|<C
$$

If $a<1 / 3$, we can again use contour integration but in $\{\Im \xi<0, \Re \xi>0\}$. The contour should again be taken as the union of semicircles. We are left with $a \sim 1 / 2$ case. If $a>1 / 2$, then, 
again, use contour integration where $\gamma_{1(3)}$ are the same but $\gamma_{2}$ is the union of arcs on the circles $|\xi-2 a|=a$ and $|\xi|=1$. Then,

$$
\sup _{1>a>1 / 2, T>0}\left|\int_{a}^{3 a} \frac{\exp \left(i T\left(\xi+\xi^{-1}\right)\right)}{\xi-2 a} d \xi\right| \lesssim 1+\sup _{1>a>1 / 2, T>0}\left|\int_{-\theta_{1}}^{\theta_{1}} \frac{e^{i T \cos \theta}}{2 a-e^{i \theta}} e^{i \theta} d \theta\right|
$$

where $\theta_{1}$ depends on $a$. The Taylor expansion around zero gives

$$
\begin{gathered}
\sup _{1>a>1 / 2, T>0}\left|\int_{-\theta_{1}}^{\theta_{1}} \frac{e^{i T \cos \theta}}{2 a-e^{i \theta}} e^{i \theta} d \theta\right| \lesssim 1+\sup _{a>1 / 2, T>0}\left|\int_{-\theta_{1}}^{\theta_{1}} \frac{e^{i T \cos \theta}}{2 a-1-i \theta} d \theta\right| \\
\lesssim 1+\int_{-\theta_{1}}^{\theta_{1}} \frac{2 a-1}{(2 a-1)^{2}+\theta^{2}} d \theta<C
\end{gathered}
$$

by canceling the odd part. The case $a<1 / 2$ is identical and the calculation for $a=1 / 2$ follows from the similar contour integration and the bound

$$
\sup _{\delta>0, T>0}\left|\int_{\delta<|\theta|<\theta_{1}} \frac{e^{i T \cos \theta}}{1-e^{i \theta}} e^{i \theta} d \theta\right|<C
$$

\section{Acknowledgements}

This research was supported by Alfred P. Sloan Research Fellowship and NSF Grant DMS-0758239.

\section{References}

[1] M. Christ, A. Kiselev. Scattering and wave operators for one-dimensional Schrödinger operators with slowly decaying nonsmooth potentials. Geom. Funct. Anal., 12 (2002), 1174-1234.

[2] D. Damanik, B. Simon. Jost functions and Jost solutions for Jacobi matrices. I. A necessary and sufficient condition for Szegö asymptotics. Invent. Math., 165 (2006), No. 1, 1-50.

[3] S. Denisov. On weak asymptotics for Schrödinger evolution. Mathematical Modelling of Natural Phenomena (to appear).

[4] S. Denisov. On the existence of wave operators for some Dirac operators with square summable potential. Geom. Funct. Anal., 14 (2004), No. 3, 529-534.

[5] S. Denisov, S. Kupin. Asymptotics of the orthogonal polynomials for the Szegó class with a polynomial weight. J. Approx. Theory, 139 (2006), No. 1-2, 8-28. 
[6] S. Denisov. Absolutely continuous spectrum of multidimensional Schrödinger operator. Int. Math. Res. Not., 2004, No. 74, 3963-3982.

[7] R. Killip. Perturbations of one-dimensional Schrödinger operators preserving the absolutely continuous spectrum. Int. Math. Res. Not., 2002, 2029-2061.

[8] R. Killip, B. Simon. Sum rules and spectral measure of Schrödinger operators with $L^{2}$ potentials. Ann. of Math., (2) 170 (2009), No. 2, 739-782.

[9] P. Lax, R. Phillips. Scattering theory. Pure and Applied Mathematics, Academic Press Inc., Boston, 1989.

[10] S.N. Naboko. Dense point spectra of Schrödinger and Dirac operators. Theor. Mat. Fiz., 68 (1986), 18-28.

[11] B. Simon. Orthogonal polynomials on the unit circle. Parts 1 and 2. American Mathematical Society Colloquium Publications, American Mathematical Society, Providence, 2005.

[12] B. Simon. Some Schrödinger operators with dense point spectrum. Proc. Amer. Math. Soc., 125 (1997), 203-208. 